\title{
Edge magnetoplasmons in periodically modulated structures
}

\author{
O. G. Balev, ${ }^{a, b}$ Nelson Studart, ${ }^{a}$ and P. Vasilopoulos ${ }^{c}$ \\ ${ }^{a}$ Departmento de Fisica, Universidade Federal de São Carlos, 13565-905 São Carlos, SP, Brazil \\ ${ }^{b}$ Institute of Physics of Semiconductors, National Academy of Sciences, 45 Pr. Nauky, Kiev 252650, Ukraine \\ ${ }^{c}$ Concordia University, Department of Physics, 1455 de Maisonneuve Blvd O, Montréal, Québec, Canada, H3G 1M8
}

We present a microscopic treatment of edge magnetoplasmons (EMP's) within the random-phase approximation for strong magnetic fields, low temperatures, and filling factor $\nu=1(2)$, when a weak short-period superlattice potential is imposed along the Hall bar. The modulation potential modifies both the spatial structure and the dispersion relation of the fundamental EMP and leads to the appearance of a novel gapless mode of the fundamental EMP. For sufficiently weak modulation strengths the phase velocity of this novel mode is almost the same as the group velocity of the edge states but it should be quite smaller for stronger modulation. We discuss in detail the spatial structure of the charge density of the renormalized and the novel fundamental EMP's.

PACS 73.20.Dx, 73.20.Mf; 73.40.Hm

\section{INTRODUCTION}

The theory of edge magnetoplasmons (EMP's) plays a fundamental role in the quantum Hall effect (QHE) due to a close interplay with time-resolved transport experiments. [1] Classical [2] and quantum [3] models have been used to describe EMP modes through different wave mechanisms at the edges of the two-dimensional electron system (2DES). Recently a quasi-microscopic description for EMP's in the QHE regime, embracing the edge-wave mechanisms mentioned above, has been proposed [4] that takes into account the lateral confining potential, the structure of the Landau levels (LL) for integer values of the filling factor $\nu$, and the dissipation which conditions the propagation of the modes. The lateral confining potential is flat in the interior of the channel and smooth on the scale of the magnetic length $\ell_{0}$ but sufficiently steep that the Landau level (LL) flattening can be neglected. The theoretical framework was also extended to take into account nonlocal contributions to the current density within the randomphase approximation. [5]

There has been a great number of studies of magnetotransport properties of 2DES's modulated by one-dimensional (1D) lateral superlattices with large period $a \gtrsim 100 \mathrm{~nm}$. [6.6] The spectrum of magnetoplasmon excitations has been studied in such systems as well [8]. Also, many works have been devoted to magnetotransport and related phenomena in the case of 2D lateral superlattices both in the regime of relatively weak modulation [9, 10 and for antidot arrays [11], where the 2DES cannot penetrate into the antidot region with higher potential. Quite recently a superlattice field effect transistor was designed in which the 2DES in a GaAs-based sample is subjected to a atomically precise $1 \mathrm{D}$ potential with period of $15 \mathrm{~nm}$ [12] and vicinal superlattices were produced with $a \approx 16 \mathrm{~nm}$. 13] More recently, attention has been focused on transport of commensurate composite fermions in weak periodic electrostatic potentials at the half-filled LL. [7]

In this work we employ the self-consistent field formalism, or random-phase approximation (RPA), to study the effect of a 1D weak periodic modulation, with period $a$, on the fundamental EMPs in the quantum Hall system for LL filling factors $\nu=1(2)$ and low temperatures, $k_{B} T \ll \hbar v_{g} / \ell_{0}$, where $v_{g}$ is the group velocity of edge states. Motivated by recent results on the 2DES subjected to lateral superlattice potentials with short period 12. 13], we restrict our study, starting from Sec. III, to the short-period regime $a \ll 2 \pi \ell_{0}$. More precisely we will assume $\exp \left[-\left(\pi \ell_{0} / a\right)^{2}\right] \ll 1$.

In Sec. II we derive the integral equations for the wave charge density and electrostatic potential at the edge of a periodically modulated channel. In Sec. III, we present our result for the dispersion relations and spatial structures of the fundamental EMPs. Finally, in Sec. IV we summarize our major conclusions.

\section{INTEGRAL EQUATIONS FOR EMPS}

The non-interacting zero-thickness 2DEG, of width $W$ and length $L_{x}=L$, in the presence of a strong magnetic field $B$ along the $z$ axis and under a 1D periodic modulation, is described by the Hamiltonian $\hat{H}_{0}=\hat{h}^{0}+V_{s}(x)$, where $\hat{h}^{0}=\left[\left(\hat{p}_{x}+e B y / c\right)^{2}+\hat{p}_{y}^{2}\right] / 2 m^{*}+V_{y}$. The confining potential is flat in the interior of the 2DES, $\left(V_{y}=0\right)$ and parabolic at its edges, $V_{y}=m^{*} \Omega^{2}\left(y-y_{r}\right)^{2} / 2, y \geq y_{r}$. We assume that $V_{y}$ is smooth on the scale of $\ell_{0}$ such that $\Omega \ll \omega_{c}$, where $\omega_{c}=|e| B / m^{*} c$ is the cyclotron frequency. The $1 \mathrm{D}$ modulation $V_{s}(x)=V_{s} \cos (G x)$ is a weak periodic potential 
with $G=2 \pi / a$. Within the RPA framework, the corresponding one-electron density matrix $\hat{\rho}$ obeys the equation of motion

$$
i \hbar \frac{\partial \hat{\rho}}{\partial t}=[\hat{H}(t), \hat{\rho}]-\frac{i \hbar}{\tau}\left(\hat{\rho}-\hat{\rho}^{(0)}\right)
$$

where $\hat{H}(t)=\hat{H}_{0}+V(x, y, t)$ with

$$
V(x, y, t)=e^{-i\left(\omega_{0} t-q_{x} x\right)} \sum_{l=-\infty}^{\infty} V_{l}\left(\omega_{0}, q_{x}, y\right) e^{i G l x}+\text { c.c. }
$$

Without interaction the one-electron density matrix $\hat{\rho}^{(0)}$ is diagonal, i.e., $\left\langle\alpha\left|\hat{\rho}^{(0)}\right| \beta>=f_{\alpha} \delta_{\alpha \beta}\right.$, where $f_{\alpha}=[1+$ $\left.\exp \left(\left(E_{\alpha}-E_{F}\right) / k_{B} T\right)\right]^{-1}$ is the Fermi-Dirac function; $\hat{H}_{0}\left|\alpha>=E_{\alpha}\right| \alpha>$. Notice that $\tau \rightarrow \infty$ corresponds to the collisionless case while a finite $\tau$ provides the possibility of estimating roughly the influence of collisions. Both $\hat{H}_{0}$ and the sum $\sum_{l=-\infty}^{\infty} V_{l}\left(\omega_{0}, q_{x}, y\right) e^{i G l x}$ are periodic along $x$ with period $a$.

Equation (11) can be solved by Laplace transforms. [5] Taking the trace of $\hat{\rho}$ with the electron density operator $e \delta(\mathbf{r}-\hat{\mathbf{r}})$ gives the wave charge density in the form

$$
\rho\left(\omega_{0}, x, y\right)=e^{i q_{x} x} \sum_{l=-\infty}^{\infty} \rho_{l}\left(\omega_{0}, q_{x}, y\right) e^{i G l x} .
$$

The charge density $\rho_{l}\left(\omega, q_{x}, y\right) \exp \left[i\left(q_{x}+G l\right) x\right]$ induces a wave electric potential $\phi_{l}\left(\omega, q_{x}, y\right) \exp \left[i\left(q_{x}+G l\right) x\right]$. From Poisson's equation this is given as

$$
\phi_{l}\left(\omega, q_{x}, y\right)=\frac{2}{\epsilon} \int_{-\infty}^{\infty} d y^{\prime} K_{0}\left(\left|q_{x}+G l\right|\left|y-y^{\prime}\right|\right) \times \rho_{l}\left(\omega, q_{x}, y^{\prime}\right)
$$

where $\epsilon$ is the background dielectric constant, assumed spatially homogeneous, and $K_{0}(x)$ is the modified Bessel function; $\phi$ and $\rho$ pertain to the $2 \mathrm{D}$ plane. Taking $\left|q_{x}\right| W \gg 1$, we can consider an EMP along the right edge of the channel of the form $A\left(\omega, q_{x}, x, y\right) \exp \left[-i\left(\omega t-q_{x} x\right)\right]$ totally independent of the left edge, where $A\left(\omega, q_{x}, x, y\right)$ is periodic along $x$ with period $a$.

In the absence of an external potential $V_{l}\left(\omega, q_{x}, y\right)=e \phi_{l}\left(\omega, q_{x}, y\right)$. As a result, considering large time responses, $t \gg \tau$, after some straightforward calculations we obtain the following integral equation for $\rho_{m}\left(\omega, q_{x}, y\right)$

$$
\begin{aligned}
\rho_{m}\left(\omega, q_{x}, y\right)= & \frac{2 e^{2}}{\epsilon L} \sum_{\alpha, \beta} \sum_{l=-\infty}^{\infty} \int_{0}^{L} d x e^{-i\left(q_{x}+G m\right) x} \psi_{\beta}^{*}(\mathbf{r}) \psi_{\alpha}(\mathbf{r}) \\
& \times \frac{f_{\beta}-f_{\alpha}}{E_{\beta}-E_{\alpha}+\hbar \omega+i \hbar / \tau} \int d \tilde{\mathbf{r}} e^{i\left(q_{x}+G l\right) \tilde{x}} \psi_{\alpha}^{*}(\tilde{\mathbf{r}}) \psi_{\beta}(\tilde{\mathbf{r}}) \\
& \times \int_{-\infty}^{\infty} d y^{\prime} K_{0}\left(\left|q_{x}+G l\right|\left|\tilde{y}-y^{\prime}\right|\right) \rho_{l}\left(\omega, q_{x}, y^{\prime}\right),
\end{aligned}
$$

where $\psi_{\alpha}=\langle\mathbf{r} \mid \alpha\rangle$ and we dropped the subscript 0 from $\omega_{0}$. For definiteness, we take $\omega>0$.

We consider low temperatures $T$ satisfying $\hbar v_{g n} \gg \ell_{0} k_{B} T$, where $v_{g n}$ is the group velocity of the edge states of $n$-th LL. Furthermore we will assume the long-wavelength limit $q_{x} \ell_{0} \ll 1$, which is well satisfied, e.g., for the fundamental EMP in the low-frequency regime, $\omega \ll \omega_{c}$. 狛 Then, assuming that the condition $G v_{g n} \ll \omega_{c}$ is satisfied and comparing the terms proportional to $f_{\beta^{*}}$, for a given $n_{\beta^{*}}$, of the right-hand side (RHS) of Eq. (5), we conclude that the contribution to the summation over $n_{\alpha}$ with $n_{\alpha}=n_{\beta^{*}}$ is much larger than any other term of this sum or the sum of all terms with $n_{\alpha} \neq n_{\beta^{*}}$. The small parameter is $\left|\omega-q_{x} v_{g n_{\beta^{*}}}\left(k_{x \beta}\right)\right| / \omega_{c} \ll 1$, where $v_{g n_{\beta^{*}}}\left(k_{x \beta}\right)$ is the group velocity of an occupied state $\left|n_{\beta^{*}}, k_{x \beta}\right\rangle$ of the $n_{\beta^{*}}$ LL. The inequality above also implies that $q_{x} v_{g 0} / \omega_{c} \ll 1$, since $v_{g 0}$ has typically the largest value among $v_{g n}$. Similar results follow from an analysis of the terms proportional to $f_{\alpha^{*}}$ in the summation over $n_{\beta}$ on the RHS of Eq. (5). Hence, for $\omega \ll \omega_{c}, q_{x} v_{g 0} \ll \omega_{c}$ and $G v_{g 0} \ll \omega_{c}$, the terms with $n_{\alpha} \neq n_{\beta}$ can be neglected. Then the integral equation for the electron charge density $\rho_{m}\left(\omega, q_{x}, y\right)$ becomes

$$
\rho_{m}\left(\omega, q_{x}, y\right)=\frac{e^{2}}{L} \sum_{n_{\alpha}=0}^{\bar{n}} \sum_{k_{x \alpha}} \sum_{k_{x \beta}} \sum_{l=-\infty}^{\infty} \int_{0}^{L} d x e^{-i\left(q_{x}+G m\right) x} \psi_{n_{\alpha} k_{x \beta}}^{*}(\mathbf{r}) \psi_{n_{\alpha} k_{x \alpha}}(\mathbf{r})
$$




$$
\begin{aligned}
& \times \frac{f_{n_{\alpha} k_{x \beta}}-f_{n_{\alpha} k_{x \alpha}}}{E_{n_{\alpha} k_{x \beta}}-E_{n_{\alpha} k_{x \alpha}}+\hbar \omega+i \hbar / \tau} \\
& \times \int d \tilde{\mathbf{r}} e^{i\left(q_{x}+G l\right) \tilde{x}} \psi_{n_{\alpha} k_{x \alpha}}^{*}(\tilde{\mathbf{r}}) \psi_{n_{\alpha} k_{x \beta}}(\tilde{\mathbf{r}}) \phi_{l}\left(\omega, q_{x}, \tilde{y}\right),
\end{aligned}
$$

where $\bar{n}$ denotes the highest occupied Landau level (LL). For even $\nu$, the (RHS) of Eq. (6) should be multiplied by 2 , the spin degeneracy factor; for $\nu$ even the spin-splitting is neglected. Equation (6), for $m=0, \pm 1, \pm 2, \ldots$, gives a system of integral equations, whose solution determines $\rho_{m}\left(\omega, q_{x}, y\right)$ in the RHS of Eq. (3).

Because $V_{s}(x)$ is assumed weak, the eigenfunctions $\psi_{n_{\alpha} k_{x \alpha}}=\left\langle\mathbf{r} \mid n_{\alpha}, k_{x \alpha}\right\rangle$ and the eigenvalues $E_{n_{\alpha} k_{x \alpha}}$ of $\hat{H}_{0}$ can be evaluated by second-order perturbation theory. A straightforward calculation leads to [14]

$$
\begin{aligned}
\left|n_{\alpha}, k_{x \alpha}\right\rangle= & {\left[1-\tilde{V}_{s n_{\alpha}}^{2}\left(k_{x \alpha}\right)\right]\left|n_{\alpha}, k_{x \alpha}\right\rangle^{(0)}+\tilde{V}_{s n_{\alpha}}\left(k_{x \alpha}\right)\left[\left|n_{\alpha}, k_{x \alpha}-G\right\rangle^{(0)}-\left|n_{\alpha}, k_{x \alpha}+G\right\rangle^{(0)}\right] } \\
& +\tilde{V}_{s n_{\alpha}}^{2}\left(k_{x \alpha}\right)\left[\left|n_{\alpha}, k_{x \alpha}+2 G\right\rangle^{(0)}+\left|n_{\alpha}, k_{x \alpha}-2 G\right\rangle^{(0)}\right] .
\end{aligned}
$$

Here we have introduced a dimensionless parameter $\tilde{V}_{s n}\left(k_{x}\right)$ characterizing the strength of the periodic potential for the $n$-th LL, near its edge, and given by

$$
\tilde{V}_{s n}\left(k_{x}\right)=\frac{V_{s}}{2 \hbar G v_{g n}\left(k_{x}\right)} e^{-\left(G \ell_{0} / 2\right)^{2}} L_{n}\left(\left(G \ell_{0}\right)^{2} / 2\right)
$$

where $v_{g n}\left(k_{x}\right)=\hbar^{-1} \partial E_{n}\left(k_{x}\right) / \partial k_{x}$ is the group velocity of a state in the edge region of the $n$-th LL and $L_{n}(x)$ is the Laguerre polynomial. Due to the smoothness of the confining potential on the $\ell_{0}$ scale, the unperturbed eigenfunctions are well approximated by $\psi_{n_{\alpha} k_{x \alpha}}^{(0)} \equiv\left\langle\mathbf{r} \mid n_{\alpha}, k_{x \alpha}\right\rangle^{(0)} \equiv \psi_{\alpha}^{(0)}(\mathbf{r}) \approx e^{i k_{x} x} \Psi_{n}\left(y-y_{0}\right) / \sqrt{L}$, where $\Psi_{n}(y)$ is the harmonic oscillator function. Because we have used the condition $\omega_{c} \gg G v_{g n}$ to obtain Eq. (7), the small "nonresonance" contributions with $n_{\beta} \neq n_{\alpha}$ can be neglected. In the edge region the evaluation of the eigenvalue $E_{n_{\alpha} k_{x \alpha}} \equiv E_{n_{\alpha}}\left(k_{x \alpha}\right)$, by perturbation theory, shows that the first-order correction $E_{n_{\alpha}}^{(1)}\left(k_{x \alpha}\right)$ vanishes. As for the second-order correction $E_{n_{\alpha}}^{(2)}\left(k_{x \alpha}\right)$, the main "resonance" contributions to it, with $n_{\beta}=n_{\alpha}$, are mutually cancelled due to imposed conditions. Then it can be shown that $E_{n_{\alpha}}\left(k_{x \alpha}\right)$ in the edge region can be well approximated by the zero-order term, i.e., $E_{n_{\alpha}}\left(k_{x \alpha}\right) \approx E_{n_{\alpha}}^{(0)}\left(k_{x \alpha}\right)$. The energy spectrum of the $n$-th LL, $E_{\alpha}^{(0)} \approx(n+1 / 2) \hbar \omega_{c}+m^{*} \Omega^{2}\left(y_{0}-y_{r}\right)^{2} / 2$, leads to the group velocity of the edge states $v_{g n}=\partial E_{n}\left(k_{r}+k_{e}^{(n)}\right) / \hbar \partial k_{x}=\hbar \Omega^{2} k_{e}^{(n)} / m^{*} \omega_{c}^{2}$ with characteristic wave vector $k_{e}^{(n)}=\left(\omega_{c} / \hbar \Omega\right) \sqrt{2 m^{*} \Delta_{F n}}, \Delta_{F n}=E_{F}-(n+1 / 2) \hbar \omega_{c}$, where $E_{F}$ is the Fermi energy. The edge of the $n$-th LL is denoted by $y_{r n}=y_{r}+\ell_{0}^{2} k_{e}^{(n)}=\ell_{0}^{2} k_{r n}$, where $k_{r n}=k_{r}+k_{e}^{(n)}$, and $W=2 y_{r 0}$. We can also write $v_{g n}=c E_{e n} / B$, where $E_{e n}=\Omega \sqrt{2 m^{*} \Delta_{F n}} /|e|$ is the electric field associated with the confining potential $V_{y}$ at $y_{r n}$. We have also introduced the wave vector $k_{r}=y_{r} / \ell_{0}^{2}$. The typical width of the edge region for the $n$-th LL can be estimated here as $\eta \ell_{0}^{2} k_{e}^{(n)} \gg \ell_{0}$, where $\eta \ll 1$. For all occupied LLs, we assume that $k_{e}^{(n)} \gg G \gg 1 / \ell_{0}$. Since in Eqs. (5) and (6) the significant eigenstates are localized along the $y$ direction near the right edge of the channel, i.e., with $y_{0}\left(k_{x}\right)>y_{r}$, we have considered only these eigenstates in Eqs. (7) and (8). Moreover, it follows from Eq. (6) that the main contributions come from $k_{x} \approx k_{r n}$ and $k_{x} \approx\left(k_{r n} \pm G\right)$; thus for the applicability of the perturbation theory here it is sufficient to assume that $\tilde{V}_{s n}\left(k_{r n}\right) \equiv \tilde{V}_{s n} \ll 1$.

\section{FUNDAMENTAL EMPS FOR $\nu=1(2)$}

We first consider the case $\nu=1$ and then indicate how the results change for $\nu=2$. For $\nu=1$, we have $\bar{n}=0$ in Eq. (6). We will look for gapless edge modes, with $\omega \rightarrow 0$ for $q_{x} \rightarrow 0$, and assume that $1 \gg \tilde{V}_{s 0} \gtrsim \exp \left[-\left(G \ell_{0} / 2\right)^{2}\right]$, where $\tilde{V}_{s 0}=\left(V_{s} / 2 \hbar G v_{g 0}\right) \exp \left[-\left(G \ell_{0} / 2\right)^{2}\right]$, which implies the short-period regime, $G \ell_{0} \gg 1$. ¿From Eq. (6) for $m=0$, we can write the integral equation for $\rho_{0}\left(\omega, q_{x}, y\right)$ in the form

$$
\rho_{0}\left(\omega, q_{x}, y\right)=\left[\hat{F}_{1}+\hat{F}_{2}\right] \int_{-\infty}^{\infty} d y^{\prime} K_{0}\left(\left|q_{x} \| \tilde{y}-y^{\prime}\right|\right) \rho_{0}\left(\omega, q_{x}, y^{\prime}\right),
$$

where the integral functional $\hat{F}_{1}$ is given as 


$$
\begin{aligned}
\hat{F}_{1}= & \frac{e^{2}}{\pi \hbar \epsilon} \int_{-\infty}^{\infty} d k_{x \alpha} \Pi\left(y, k_{x \alpha}\right) \frac{f_{0, k_{x \alpha}-q_{x}}-f_{0, k_{x \alpha}}}{\omega-v_{g 0}\left(k_{x \alpha}\right) q_{x}+i / \tau} \\
& \times \int_{-\infty}^{\infty} d \tilde{y}\left\{\Pi\left(\tilde{y}, k_{x \alpha}\right)+\tilde{V}_{s 0}^{2}\left[\Pi\left(\tilde{y}, k_{x \alpha}-G\right)+\Pi\left(\tilde{y}, k_{x \alpha}+G\right)\right]\right\},
\end{aligned}
$$

with $\Pi\left(y, k_{x \alpha}\right)=\left|\Psi_{0}\left(y-y_{0}\left(k_{x \alpha}\right)\right)\right|^{2}$. The integral functional $\hat{F}_{2}$ is given as

$$
\begin{aligned}
\hat{F}_{2}= & \frac{e^{2}}{\pi \hbar \epsilon} \int_{-\infty}^{\infty} d k_{x \alpha} \tilde{V}_{s 0}^{2}\left[\Pi\left(y, k_{x \alpha}-G\right)+\Pi\left(y, k_{x \alpha}+G\right)\right] \\
& \times \frac{f_{0, k_{x \alpha}-q_{x}}-f_{0, k_{x \alpha}}}{\omega-v_{g 0}\left(k_{x \alpha}\right) q_{x}+i / \tau} \int_{-\infty}^{\infty} d \tilde{y} \Pi\left(\tilde{y}, k_{x \alpha}\right) .
\end{aligned}
$$

In Eqs. (9)-(11), for $q_{x} \rightarrow 0$, we can make approximation $\left(f_{0, k_{x \alpha}-q_{x}}-f_{0, k_{x \alpha}}\right) \approx q_{x} \delta\left(k_{x \alpha}-k_{r 0}\right)$. After integration over $k_{x \alpha}$, we obtain

$$
\begin{aligned}
\rho_{0}\left(\omega, q_{x}, \bar{y}\right)= & \frac{e^{2}}{\pi \hbar \epsilon} \frac{q_{x}}{\tilde{\omega}}\left\{\Psi_{0}^{2}(\bar{y}) \int_{-\infty}^{\infty} d \bar{y}^{\prime}\left[\Psi_{0}^{2}\left(\bar{y}^{\prime}\right)+\tilde{V}_{s 0}^{2}\left[\Psi_{0}^{2}\left(\bar{y}^{\prime}+G \ell_{0}^{2}\right)+\Psi_{0}^{2}\left(\bar{y}^{\prime}-G \ell_{0}^{2}\right)\right]\right]\right. \\
& \left.+\tilde{V}_{s 0}^{2}\left[\Psi_{0}^{2}\left(\bar{y}+G \ell_{0}^{2}\right)+\Psi_{0}^{2}\left(\bar{y}-G \ell_{0}^{2}\right)\right] \int_{-\infty}^{\infty} d \bar{y}^{\prime} \Psi_{0}^{2}\left(\bar{y}^{\prime}\right)\right\} \\
& \times \int_{-\infty}^{\infty} d \bar{y}^{\prime \prime} K_{0}\left(\left|q_{x} \| \bar{y}^{\prime}-\bar{y}^{\prime \prime}\right|\right) \rho_{0}\left(\omega, q_{x}, \bar{y}^{\prime \prime}\right),
\end{aligned}
$$

where $\bar{y}=y-y_{r 0}$, and $\tilde{\omega}=\omega-q_{x} v_{g 0}+i / \tau$. In order to simplify the notation we take $\rho_{i}\left(\omega, q_{x}, y\right) \equiv \rho_{i}\left(\omega, q_{x}, \bar{y}\right)$, $i=0, \pm 1, \ldots$

Similarly, omitting minor terms in Eq. (6) we obtain, for $m=1$,

$$
\begin{aligned}
\rho_{1}\left(\omega, q_{x}, \bar{y}\right)= & \frac{e^{2}}{\pi \hbar \epsilon} \tilde{V}_{s 0} \frac{q_{x}}{\tilde{\omega}} \Psi_{0}(\bar{y})\left[\Psi_{0}\left(\bar{y}+G \ell_{0}^{2}\right)-\Psi_{0}\left(\bar{y}-G \ell_{0}^{2}\right)\right] \\
& \times \int_{-\infty}^{\infty} d \tilde{y} \Psi_{0}^{2}(\tilde{y}) \int_{-\infty}^{\infty} d \bar{y}^{\prime} K_{0}\left(\left|q_{x}\right|\left|\tilde{y}-\bar{y}^{\prime}\right|\right) \rho_{0}\left(\omega, q_{x}, \bar{y}^{\prime}\right) .
\end{aligned}
$$

From Eq. (6) we find, for $m=-1, \rho_{-1}\left(\omega, q_{x}, y\right) \equiv \rho_{1}\left(\omega, q_{x}, y\right)$.

The general solution of the linear homogeneous integral equation, Eq. (12), can be sought in the form

$$
\rho_{0}\left(\omega, q_{x}, y\right)=\rho_{0}^{(0)}\left(\omega, q_{x}\right) \Psi_{0}^{2}(\bar{y})+\rho_{0}^{(1)}\left(\omega, q_{x}\right)\left[\Psi_{0}^{2}\left(\bar{y}+G \ell_{0}^{2}\right)+\Psi_{0}^{2}\left(\bar{y}-G \ell_{0}^{2}\right)\right] .
$$

Substituting Eq. (14) into Eq. (12) and equating the coefficients of $\Psi_{0}^{2}(\bar{y})$ and $\left[\Psi_{0}^{2}\left(\bar{y}+G \ell_{0}^{2}\right)+\Psi_{0}^{2}\left(\bar{y}-G \ell_{0}^{2}\right)\right]$ on both sides of Eq. (12), we obtain two linear homogeneous equations for $\rho_{0}^{(i)}\left(\omega, q_{x}\right), i=0,1$ :

$$
\begin{aligned}
\rho_{0}^{(0)}= & \frac{e^{2}}{\pi \hbar \epsilon} \frac{q_{x}}{\tilde{\omega}}\left\{\left[a_{00}\left(q_{x}\right)+2 \tilde{V}_{s 0}^{2} a_{00}^{00}\left(q_{x}, G \ell_{0}^{2}\right)\right] \rho_{0}^{(0)}\right. \\
& \left.+2\left[a_{00}^{00}\left(q_{x}, G \ell_{0}^{2}\right)+\tilde{V}_{s 0}^{2}\left(a_{00}\left(q_{x}\right)+a_{00}^{00}\left(q_{x}, 2 G \ell_{0}^{2}\right)\right)\right] \rho_{0}^{(1)}\right\}
\end{aligned}
$$

and

$$
\rho_{0}^{(1)}=\frac{e^{2}}{\pi \hbar \epsilon} \tilde{V}_{s 0}^{2} \frac{q_{x}}{\tilde{\omega}}\left\{a_{00}\left(q_{x}\right) \rho_{0}^{(0)}+2 a_{00}^{00}\left(q_{x}, G \ell_{0}^{2}\right) \rho_{0}^{(1)}\right\},
$$

where the coefficients $a_{n n}^{m m}$ are given by [5]

$$
a_{n n}^{m m}\left(q_{x}, \Delta y\right)=\int_{-\infty}^{\infty} \int_{-\infty}^{\infty} d x d x^{\prime} \Psi_{n}^{2}(x) \Psi_{m}^{2}\left(x^{\prime}\right) K_{0}\left(\left|q_{x}\right|\left|x-x^{\prime}+\Delta y\right|\right)
$$

Here $a_{n n}^{m m}\left(q_{x}, \Delta y\right)=a_{m m}^{n n}\left(q_{x}, \Delta y\right), a_{n n}^{m m}\left(q_{x}, \Delta y\right)=a_{n n}^{m m}\left(q_{x},-\Delta y\right)$ and $a_{00}^{00}\left(q_{x}, 0\right)=a_{00}\left(q_{x}\right)$. We will assume $2 \pi q_{x} \ell_{0}^{2} / a \ll 1$. Notice that $a_{00}\left(q_{x}\right) \approx \ln \left(1 / q_{x} \ell_{0}\right)+3 / 4$ and, for $\Delta y / \ell_{0} \gg 1, a_{00}^{00}\left(q_{x}, \Delta y\right) \approx \ln \left(2 / q_{x} \ell_{0}\right)-\gamma-\ln \left(\Delta y / \ell_{0}\right) \approx$ $\ln \left(1 / q_{x} \Delta y\right)+0.1$, where $\gamma$ is the Euler constant. 


\section{A. Dispersion Relations}

The dimensionless frequencies $\omega^{\prime}=\tilde{\omega} /\left(e^{2} q_{x} / \pi \hbar \epsilon\right)$ of the branches resulting from the determinantal solution of the two linear homogeneous equations for $\rho_{0}^{(i)}\left(\omega, q_{x}\right)$, Eqs. (15) and (16), are given by

$$
\begin{aligned}
\omega_{ \pm}^{\prime}= & \frac{1}{2} a_{00}\left(q_{x}\right)+2 \tilde{V}_{s 0}^{2} a_{00}^{00}\left(q_{x}, G \ell_{0}^{2}\right) \pm \frac{1}{2} a_{00}\left(q_{x}\right) \\
& \times\left\{1+8 \tilde{V}_{s 0}^{2} a_{00}^{-1}\left(q_{x}\right)\left[a_{00}^{00}\left(q_{x}, G \ell_{0}^{2}\right)+\tilde{V}_{s 0}^{2}\left(a_{00}\left(q_{x}\right)+a_{00}^{00}\left(q_{x}, 2 G \ell_{0}^{2}\right)\right)\right]\right\}^{1 / 2} .
\end{aligned}
$$

From Eq. (18), it follows that the effect of the modulation potential on the fundamental EMP is quite strong. Apart from the renormalization of the fundamental EMP of $n=0 \mathrm{LL}$ with dispersion

$$
\omega_{+}^{\prime} \approx a_{00}\left(q_{x}\right)+4 \tilde{V}_{s 0}^{2} a_{00}^{00}\left(q_{x}, G \ell_{0}^{2}\right)
$$

it leads to the existence of a novel fundamental EMP of $n=0 \mathrm{LL}$ with dispersion

$$
\omega_{-}^{\prime} \approx 2 \tilde{V}_{s 0}^{4} a_{00}^{-1}\left(q_{x}\right)\left\{2\left[a_{00}^{00}\left(q_{x}, G \ell_{0}^{2}\right)\right]^{2}-a_{00}\left(q_{x}\right)\left[a_{00}\left(q_{x}\right)+a_{00}^{00}\left(q_{x}, 2 G \ell_{0}^{2}\right)\right]\right\} .
$$

Substituting the coefficients into Eq.(20), we obtain the dispersion relation (DR) of the novel fundamental EMP

$$
\omega_{-} \approx\left[v_{g 0}-\frac{12}{\epsilon} \tilde{V}_{s 0}^{4} \sigma_{y x}^{0} \ln \left(G \ell_{0}\right)\right] q_{x}-i / \tau,
$$

where $\sigma_{y x}^{0}=\nu e^{2} / 2 \pi \hbar$ and $\nu=1(2)$. Note that for $\tilde{V}_{s 0} \leq 10^{-1}$ and $v_{g 0} \geq 10^{6} \mathrm{~cm} / \mathrm{s}$, which is a typical value in GaAs-based heterostructures, the correction in the phase velocity of this novel mode should be quite small. However, in general, e.g. for a slightly larger $\tilde{V}_{s 0}$ and a slightly smaller $v_{g 0}$, this contribution should be taken into account and can lead to a substantial decrease of the phase velocity of the novel fundamental EMP from its maximum possible value $v_{g 0}$. We point out that this contribution to Re $\omega_{-}$stems from the electron-electron interaction and the strength of the periodic modulation as well. Eq. (21) is valid for

$$
\tilde{V}_{s 0}^{2} \gg \frac{1}{3 \ln \left(G \ell_{0}\right)} \frac{\epsilon v_{g 0}}{2 \sigma_{y x}^{0}}\left(\frac{2 \pi}{k_{e}^{(0)} a}\right)^{2} .
$$

Notice that the RHS of the inequality (22) is typically very small. Under this condition, the second order correction in the group velocity $v_{g 0}\left(k_{r 0}\right)=v_{g 0}\left[1-2 \tilde{V}_{s 0}^{2}\left(2 \pi / k_{e}^{(0)} a\right)^{2}\right]$ can be neglected. As discussed above this is equivalent to neglecting $E_{0}^{(2)}\left(k_{x \alpha}\right)$.

From Eq. (19), the DR for the renormalized fundamental EMP can be written as

$$
\omega_{+} \approx q_{x} v_{g 0}+\frac{2}{\epsilon} \sigma_{y x}^{0} q_{x}\left\{\ln \left(1 / q_{x} \ell_{0}\right)+\frac{3}{4}+4 \tilde{V}_{s 0}^{2} \ln \left(1 /\left(q_{x} G \ell_{0}^{2}\right)\right)\right\}-i / \tau
$$

The term $\propto \tilde{V}_{s 0}^{2}$ shows a strong renormalization of the fundamental EMP that depends on the strength and the period of the modulation, for given value of $q_{x}$.

For a GaAs-based 2DEG and negligible dissipation, the dispersion laws for the renormalized, by the superlattice potential and intra-LL Coulomb coupling, fundamental EMP and for the novel fundamental EMP, caused by the periodic modulation $V_{s}(x)$, are shown in Fig. 1 by the top and bottom solid curves, respectively. The DRs corresponding to the $\omega_{-}$and $\omega_{+}$modes here are obtained using Eqs. (21) and (23), respectively. For the assumed parameters these equations very well approximate the exact DRs given by Eq. (18). For the sake of comparison, the dashed curve in Fig. 1 shows the fundamental EMP of $n=0 \mathrm{LL}$ in the absence of superlattice potential.

As we have discussed, the renormalization effect involves essentially intra-LL Coulomb coupling. In Fig. 1 the parameters are $m^{*} \approx 6.1 \times 10^{-29} \mathrm{~g}, \epsilon \approx 12.5$, and $\Omega \approx 7.8 \times 10^{11} \mathrm{~s}^{-1}$. [15] Assuming $\nu=1$ and $B=9 \mathrm{~T}$, these parameters lead to $\omega_{c} / \Omega \approx 30$. Here $\omega_{*}=e^{2} / \pi \hbar \epsilon \ell_{0} \approx 0.3 \omega_{c} \approx 7 \times 10^{12} \mathrm{~s}^{-1}$ is a characteristic frequency. We have also assumed $\Delta_{F 0}=\hbar \omega_{c} / 2, \tilde{V}_{s 0}=\exp (-2) \ll 1$, and $a=\pi \ell_{0} / \sqrt{2}$. This gives $v_{g 0} \approx \Omega \ell_{0} \approx 6.5 \times 10^{5} \mathrm{~cm} / \mathrm{s}, a \approx 18.5$ $\mathrm{nm}, V_{s}=2.9 \mathrm{meV}$. Observe that for these parameters, the second term in the RHS of Eq. (21) is more than 50 times smaller than the first term, $v_{g 0}$. Hence, the curve $\omega=q_{x} v_{g 0}$ will practically coincide with the solid curve at the bottom of Fig. 1.

The dispersion laws corresponding to the $\omega_{+}$and $\omega_{-}$modes, given by Eq. (18), are depicted in Fig. 2 by the top and bottom curves respectively. The solid, dot-dashed, and dashed curves correspond to $\tilde{V}_{s 0}=0.3$, 0.2 , and 0.1 , 
respectively. The data of the bottom curves were multiplied by the factor 30 . The parameters are the same as in Fig. 1 except $\Delta_{F 0}=\hbar \omega_{c} / 8, a \approx 20.6 \mathrm{~nm}$. This leads to $v_{g 0} \approx 3.25 \times 10^{5} \mathrm{~cm} / \mathrm{s}$ and to modulation strengths $V_{s} \approx 2.18 \mathrm{meV}$, $1.09 \mathrm{meV}$, and $0.73 \mathrm{meV}$ for $\tilde{V}_{s 0}=0.3,0.2$, and 0.1 , respectively. Notice that in this case $\exp \left[-\left(G \ell_{0} / 2\right)^{2}\right] \approx 0.2$. It is seen that by varying the amplitude $V_{s}$ of the periodic potential, strong modifications in the DR of the fundamental modes can occur. We observe that the phase velocity of the novel EMP decreases from its maximum value $v_{g 0}$ by increasing $\tilde{V}_{s 0}$. It can be shown that the DRs given by Eqs. (21) and (23) still represent well all curves in Fig. 2.

\section{B. Spatial Structure}

If we substitute Eq. (19) into Eq. (16), we obtain $\rho_{0}^{(1)}\left(\omega_{+}, q_{x}\right) / \rho_{0}^{(0)}\left(\omega_{+}, q_{x}\right) \approx \tilde{V}_{s 0}^{2}$. As a consequence, only a small distortion of the edge charge occurs at $\bar{y}= \pm G \ell_{0}^{2}$, in comparison with the usual one at $\bar{y}=0$. Furthermore substituting Eq. (20) in Eq. (16), we obtain $\rho_{0}^{(0)}\left(\omega_{-}, q_{x}\right) / \rho_{0}^{(1)}\left(\omega_{-}, q_{x}\right) \approx-2\left[\ln \left(1 / q_{x} \ell_{0}\right)-\ln \left(G \ell_{0}\right)\right] /\left[\ln \left(1 / q_{x} \ell_{0}\right)+3 / 4\right]>-2$. This means that the amplitude of the edge charges localized at $\bar{y}= \pm G \ell_{0}^{2}$ has absolute value approximately twice smaller in comparison with that of the charge distortion localized at $\bar{y}=0$; in addition, it has the opposite sign. Furthermore, the ratio of amplitudes of the novel fundamental EMP is independent on $V_{s 0}$ for the assumed conditions, while in the case of the renormalized fundamental EMP such ratio tends to zero. The same results hold for $\nu=2$. Thus the novel mode has a spatial structure quite different both from the spatial structure of the fundamental EMP (i.e., in the absence of modulation) and from the renormalized mode.

We proceed now to evaluate the charge density $\rho_{1}\left(\omega, q_{x}, y\right)$ induced by $\rho_{0}\left(\omega, q_{x}, y\right)$ for the two new branches: the renormalized fundamental EMP and the novel fundamental EMP. For both fundamental EMPs we obtain, from Eq. (13),

$$
\rho_{1}\left(\omega, q_{x}, y\right)=\rho_{1}\left(\omega, q_{x}\right) \Psi_{0}(\bar{y})\left[\Psi_{0}\left(\bar{y}+G \ell_{0}^{2}\right)-\Psi_{0}\left(\bar{y}-G \ell_{0}^{2}\right)\right],
$$

where, using Eq. (16), we find

$$
\rho_{1}\left(\omega, q_{x}\right)=\rho_{0}^{(1)}\left(\omega, q_{x}\right) / \tilde{V}_{s 0} .
$$

Then for the renormalized fundamental EMP the relative amplitude $\xi_{+} \equiv\left(\omega_{+}, q_{x}, y\right) / \rho_{0}\left(\omega_{+}, q_{x}, y\right) \approx$ $\tilde{V}_{s 0} \exp \left[-\left(G \ell_{0} / 2\right)^{2}\right]$, where the small factor $\exp \left[-\left(G \ell_{0} / 2\right)^{2}\right] \lesssim \tilde{V}_{s 0}$ comes from the exponentially small overlapping of the wave functions in the products of Eq. (24). Similarly, for the novel fundamental EMP, $\omega_{-}$, we obtain that

$$
\frac{\rho_{1}\left(\omega_{-}, q_{x}\right)}{\rho_{0}^{(0)}\left(\omega_{-}, q_{x}\right)} \approx-\frac{a_{00}\left(q_{x}\right)}{2 \tilde{V}_{s 0} a_{00}^{00}\left(q_{x}, G \ell_{0}^{2}\right)} \rightarrow-\frac{1}{2 \tilde{V}_{s 0}}
$$

where the limit holds for $q_{x} \rightarrow 0$. Now the relative amplitude is $\xi_{-} \equiv \rho_{1}\left(\omega_{-}, q_{x}, y\right) / \rho_{0}\left(\omega_{-}, q_{x}, y\right)=$ $\exp \left[-\left(G \ell_{0} / 2\right)^{2}\right] / 2 \tilde{V}_{s 0}$. Hence, $\xi_{-}$lies in the interval $[0.1,1]$, i.e., the amplitude of oscillations of the charge distortion $\propto \rho_{ \pm 1}\left(\omega, q_{x}, y\right)$ can be of the same order of magnitude as that $\propto \rho_{0}\left(\omega, q_{x}, y\right)$. A further treatment of Eq. (6) for $m=2$ (and $\bar{n}=0$ ) shows that the charge distortions $\rho_{2}\left(\omega, q_{x}, y\right)=\rho_{-2}\left(\omega, q_{x}, y\right)$ as compared with $\rho_{0}\left(\omega, q_{x}, y\right)$ for these two new branches have an additional small factor $\propto \tilde{V}_{s 0} \exp \left[-3\left(G \ell_{0} / 2\right)^{2}\right] \lesssim \tilde{V}_{s 0}^{4}$ with respect to the relative strength $\xi_{ \pm}$of $\rho_{ \pm 1}\left(\omega, q_{x}, y\right)$. Therefore, terms with $|l| \geq 2$ in Eq. (3) can be neglected both for the renormalized fundamental EMP and the novel fundamental EMP. As a result, from Eq. (3), we obtain straightforwardly the dimensionless form factors, $\rho_{ \pm}(x, y) \equiv \rho_{ \pm}=\sqrt{\pi} \ell_{0} \exp \left(-i q_{x} x\right) \rho\left(\omega_{ \pm}, x, y\right) / \rho_{0}^{(0)}\left(\omega_{ \pm}, q_{x}\right)$, as

$$
\begin{aligned}
\rho_{+}(x, y)= & \sqrt{\pi} \ell_{0}\left\{\Psi_{0}^{2}(\bar{y})+\tilde{V}_{s 0}^{2}\left[\Psi_{0}^{2}\left(\bar{y}+G \ell_{0}^{2}\right)+\Psi_{0}^{2}\left(\bar{y}-G \ell_{0}^{2}\right)\right]\right. \\
& \left.+2 \tilde{V}_{s 0} \cos (G x) \Psi_{0}(\bar{y})\left[\Psi_{0}\left(\bar{y}+G \ell_{0}^{2}\right)-\Psi_{0}\left(\bar{y}-G \ell_{0}^{2}\right)\right]\right\}
\end{aligned}
$$

for the renormalized fundamental mode, and

$$
\begin{aligned}
\rho_{-}(x, y)= & \sqrt{\pi} \ell_{0}\left\{\Psi_{0}^{2}(\bar{y})-\frac{1}{2}\left[\Psi_{0}^{2}\left(\bar{y}+G \ell_{0}^{2}\right)+\Psi_{0}^{2}\left(\bar{y}-G \ell_{0}^{2}\right)\right]\right. \\
& \left.-\frac{1}{\tilde{V}_{s 0}} \cos (G x) \Psi_{0}(\bar{y})\left[\Psi_{0}\left(\bar{y}+G \ell_{0}^{2}\right)-\Psi_{0}\left(\bar{y}-G \ell_{0}^{2}\right)\right]\right\}
\end{aligned}
$$


for the novel fundamental mode. In order to exhibit explicitly the $x$ dependence of the form factors, for the same parameters as used in Fig. 1, we show them in Fig. 3 for $x_{0}^{(m)}=a(m+1 / 2) / 2, m=0, \pm 1, \pm 2, \ldots$, with $\cos \left(2 \pi x_{0}^{(m)} / a\right)=$ 0 , and for $x_{1}^{(m)}=m a$, with $\cos \left(2 \pi x_{1}^{(m)} / a\right)=1$. The solid and dotted curves show $\rho_{+}(x, y)$ as a function of $Y=\bar{y} / \ell_{0}$ for $x_{1}^{(m)}=m a$ and $x_{0}^{(m)}=a(m+1 / 2) / 2$, respectively. We see that the dotted curve is exactly symmetrical with respect to $Y=0$ axis. The deviations of the solid curve from this form come from contributions to the form factor that are commensurate with the unidirectional modulation. Also in Fig. 3, $\rho_{-}(x, y)$ is shown by dot-dashed and dashed curves for $x_{1}^{(m)}$ and $x_{0}^{(m)}$, respectively. Notice that the dashed curve is symmetric while the dot-dashed curve is clearly asymmetric. In Fig. 4, we present results for the charge densities of the renormalized and novel fundamental EMPs for the same parameters that are used to obtain the solid curves in Fig. 2. That is, in Fig. 4 we use $\tilde{V}_{s 0}=0.3$ and $G \ell_{0} \approx 2.53$ in Eqs. (27) and (28).

\section{CONCLUSIONS}

We have presented a fully microscopic model for EMPs in the RPA framework valid for integer $\nu=1$ and 2 in the case of an applied 1D weak modulation $V_{s}(x)=V_{s} \cos (2 \pi x / a)$, and confining potentials that are smooth on the $\ell_{0}$ scale but still sufficiently steep at the edges that LL flattening can be neglected. [16] The model also takes into account nonlocal responses and incorporates only very weak dissipation. The main results of the present work are as follows.

i) The strength of the periodic modulation, if not too small, reshapes noticeably the spatial structure of the usual fundamental EMP of $n=0$ LL [5], normal and parallel to the edge, and substantially modifies the dispersion relation leading to a renormalized fundamental EMP of $n=0$ LL. For instance, in Fig. 1, we have seen that the group velocity of the renormalized fundamental EMP is more than $4 \%$ greater than that of the fundamental EMP without modulation for $q_{x} \ell_{0}=0.8 \times 10^{-2}$. Therefore, in time-resolved experiments, the periodic potential will imply a modulation of the propagation time of the signal due the renormalized fundamental EMP. As we have seen, this renormalization depends on the strength and period of the modulation potential.

ii) The strength of the periodic modulation, even quite small, leads to the appearance of the novel fundamental EMP with acoustical dispersion relation and phase velocity typically equal, in a GaAs-based sample, to the group velocity of the edge states, $v_{g 0}$, independent of $V_{s}$ and $a$, if $\tilde{V}_{s 0}=\left(V_{s} a / 4 \pi \hbar v_{g 0}\right) \exp \left[-\left(\pi \ell_{0} / a\right)^{2}\right] \leq 10^{-1}$ and $v_{g 0} \geq 10^{6}$ $\mathrm{cm} / \mathrm{s}$. That is, this holds for a sufficiently weak periodic modulation. However, already for $\tilde{V}_{s 0} \approx 0.2$ and $v_{g 0} \lesssim 10^{6}$ $\mathrm{cm} / \mathrm{s}$, the phase velocity of the novel fundamental EMP can be substantially smaller than $v_{g 0}$, as one can see in Fig. 2, due to the combined effect of a short-period lateral superlattice and the electron-electron interaction. In addition, its spatial structure is strongly dependent on both $\tilde{V}_{s 0}$ and $a$. The spatial structure is almost symmetric with respect to the edge of the $n=0$ LL for very weak periodic modulation. However, for not too weak $V_{s}(x)$, it becomes substantially asymmetric for some regions of $x$, as one can see by the dot-dashed curve in Figs. 3 and 4 . We have also obtained that in the latter case the contributions to the spatial structure of the novel fundamental EMP that are commensurate with the periodic modulation can be of the same order of magnitude as those that are independent of $x$.

iii) The measurement of the velocity of the novel EMP, due to its independence of the modulation parameters, in a wide range of them, can be a useful tool for obtaining directly the group velocity of edge states. Furthermore, a qualitative analysis, using results of previous studies [1] as well the above findings, shows that the dominant contribution to the damping rate of the novel EMP is absent. Then we may speculate that the damping rate of the novel mode could be rather small.

\section{ACKNOWLEDGMENTS}

This work was supported by the Brazilian FAPESP, grants no. 98/10192-2 and no. 95/0789-3, and by Canadian NSERC grant no. OGP0121756. O. G. B. thanks partial support by the Ukrainian SFFI grant no. 2.4/665 and N. S. is grateful to $\mathrm{CNPq}$ for a research fellowship. 
[1] R. C. Ashoori, H. L. Stormer, L. N. Pfeiffer, K. W. Baldwin, and K. West, Phys. Rev. B 45, 3894 (1992); N. B. Zhitenev, R. J. Haug, K. von Klitzing, and K. Eberl, Phys. Rev. Lett. 71, 2292 (1993); ibid. Phys. Rev. B 49, 7809 (1994); G. Ernst, R. J. Haug, J. Kuhl, K. von Klitzing, and K. Eberl, Phys. Rev. Lett. 77, 4245 (1996).

[2] V. A. Volkov and S. A. Mikhailov, Zh. Eksp. Teor. Fiz. 94, 217 (1988) [Sov. Phys. JETP 67, 1639 (1988)]; I. L. Aleiner and L. I. Glazman, Phys. Rev. Lett. 72, 2935 (1994).

[3] X. G. Wen, Phys. Rev. B 43, 11025 (1991); M. Stone, Ann. Phys. (NY) 207, 38 (1991); C. de Chamon and X. G. Wen, Phys. Rev. B 49, 8227 (1994); J. S. Giovanazzi, L. Pitaevskii, and S. Stringari, Phys. Rev. Lett. 72, 3230 (1994).

[4] O. G. Balev and P. Vasilopoulos, Phys. Rev. B 56, 13252 (1997); ibid. Phys. Rev. Lett. 81, 1481 (1998); O. G. Balev, P. Vasilopoulos, and Nelson Studart, J. Phys.: Condens. Matter 11, 5143(1999).

[5] O. G. Balev and P. Vasilopoulos, Phys. Rev. B 59, 2807 (1999).

[6] D. Weiss, K. von Klitzing, K. Ploog, and G. Weimann. Europhys. Lett. 8, 179 (1989); R. R. Gerhardts, D. Weiss, and K. von Klitzing, Phys. Rev. Lett. 62, 1173 (1989); R. W. Winkler, J. P. Kotthaus, and K. Ploog, Phys. Rev. Lett. 62, 1177 (1989).

[7] R. L. Willet, K. W. West, and L. N. Pfeiffer, Phys. Rev. Lett. 78, 4478 (1997); F. von Oppen, A. Stern, and B. I. Halperin, Phys. Rev. Lett. 80, 4494 (1998); S. D. M. Zwerschke and R. R. Gerhardts, Phys. Rev. Lett. 83, 2616 (1999); J. H. Smet, S. Jobst, K. von Klitzing, D. Weiss, W. Wegscheider, and V. Umansky, Phys. Rev. Lett. 83, 2620 (1999); R. L. Willet, K. W. West, and L. N. Pfeiffer, Phys. Rev. Lett. 83, 2624 (1999).

[8] H. L. Cui, V. Fessatidis, and N. J. M. Horing, Phys. Rev. Lett. 63, 2598 (1989); S. M. Stewart and C. Zhang, Semicond. Sci. Technol. 10, 1541 (1995).

[9] D. J. Thouless, M. Kohmoto, M. P. Nightingale, and M. den Nijs, Phys. Rev. Lett. 49, 405 (1982); V. Gudmundsson, R. R. Gerhardts, Phys. Rev. B 54, 5223 (1996); T. Schlosser, K. Ensslin, J. P. Kotthaus, and M. Holland, Semicond. Sci. Technol. 11, 1582 (1996).

[10] D. Pfannkuche, A. H. MacDonald, Phys. Rev. B 56, 7100 (1997); C. Albrecht, J. H. Smet, D. Weiss, K. von Klitzing, R. Henning, Phys. Rev. Lett. 83, 2234 (1999).

[11] D. Weiss, M. L. Roukes, A. Menschig, P. Grambow, K. von Klitzing, and G. Weimann, Phys. Rev. Lett. 66, 2790 (1991); S. Ishizaka and T. Ando, Phys. Rev. B 56, 15195 (1997); F. Nihey, K. Nakamura, T. Takamasu, G. Kido, T. Sakon, and M. Motokawa, Phys. Rev. B 59, 14872 (1999).

[12] R. A. Deutschamann, A. Lorke, W. Wegscheider, M. Bichler, and G. Abstreiter, Proc. of EP2DS-13, to appear in Physica E.

[13] K. Tanaka, Y. Nakamura, and H. Sakaki, Proc. of EP2DS-13, to appear in Physica E.

[14] L. D. Landau and E. M. Lifshitz, Quantum Mechanics, (Pergamon Press, New York, 1975).

[15] G. Muller, D. Weiss, A. V. Khaetskii, K, von Klitzing, S. Koch, H. Nickel, W. Schlapp, and R. Losch, Phys. Rev. B. 45, 3932 (1992).

[16] D. B. Chklovskii, B. I. Shklovskii, and L. I. Glazman, Phys. Rev. B 46, 4026 (1992); L. Brey, J. J. Palacios, and C. Tejedor, ibid 47,13884 (1993); T. Suzuki and Tsuneya Ando, J. Phys. Soc. Jpn. 62, 2986 (1993).

\section{FIGURE CAPTIONS}

Fig. 1. Dispersion relations for $\omega_{ \pm}$modes, in units of $\omega_{*}=e^{2} / \pi \hbar \epsilon \ell_{0}$, for $\nu=1$ and $B=9 \mathrm{~T}$. The upper solid curve is the renormalized fundamental EMP of the $n=0 \mathrm{LL}$ and the lower solid one is the novel fundamental EMP, due to the effect of the modulation potential. The dashed curve is the fundamental EMP in the absence of the modulation. The parameters of a GaAs-based sample are given in the text and we took $\Delta_{F 0}=\hbar \omega_{c} / 2, a \approx 18.5 \mathrm{~nm}$, and $\tilde{V}_{s 0}=\exp (-2)$.

Fig. 2. Dispersion relation of $\omega_{ \pm}$modes, in units of $\omega_{*}=e^{2} / \pi \hbar \epsilon \ell_{0}$, for values of the modulation strength $\tilde{V}_{s 0}=2.18$ $\mathrm{meV}$ (solid curve), $1.09 \mathrm{meV}$ (dot-dashed) and $0.73 \mathrm{meV}$ (dashed) and $\nu=1$ and $B=9 \mathrm{~T}$. Top (bottom) curves represent the dispersion laws for $\omega_{+}$and $\omega_{-}$modes, respectively. The values of $\omega_{-}$are multiplied by 30 . The parameters are the same as in Fig. 1 , except $\Delta_{F 0}=\hbar \omega_{c} / 8, a \approx 20.6 \mathrm{~nm}$. It follows that $v_{g 0} \approx 3.25 \times 10^{5} \mathrm{~cm} / \mathrm{s}$ and $\exp \left[-\left(G \ell_{0} / 2\right)^{2}\right] \approx 0.2$.

Fig. 3. Form factor for the fundamental EMP as a function of $Y=\bar{y} / \ell_{0}$, where $\bar{y}=y-y_{r 0}$, and $y_{r 0}$ is the edge of $n=0$ LL. The renormalized mode is indicated respectively by solid and dotted curves for $x_{1}^{(m)}=m a$ and $x_{0}^{(m)}=a(m+1 / 2) / 2$, with $m=0, \pm 1, \pm 2, \ldots$, and the novel EMP by dot-dashed and dashed curve for $x_{1}^{(m)}$ and $x_{0}^{(m)}$, respectively. The parameters used are the same as in Fig. 1.

Fig. 4. The same as in Fig. 3, but with the parameters used for plotting the solid curves in Fig. 2. 


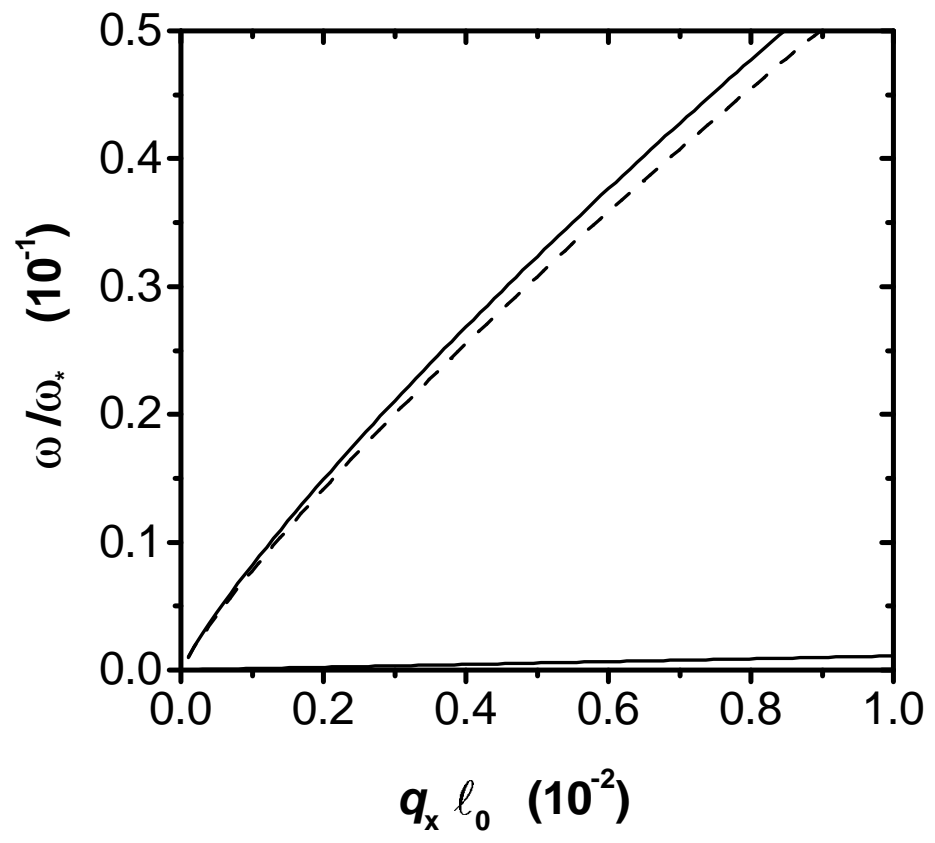

Fig. 1,

O. G. Balev et al.,

"Edge magnetoplasmons in modulated structures" 


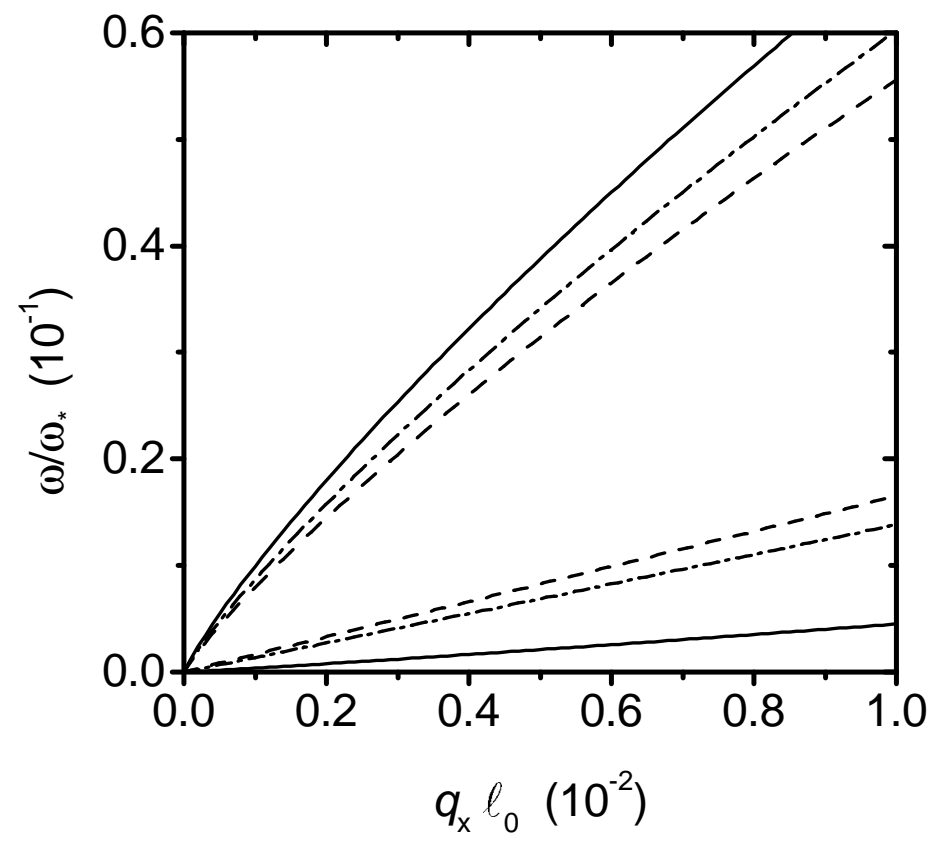

Fig. 2,

O. G. Balev et al., "Edge magnetoplasmons in modulated structures" 


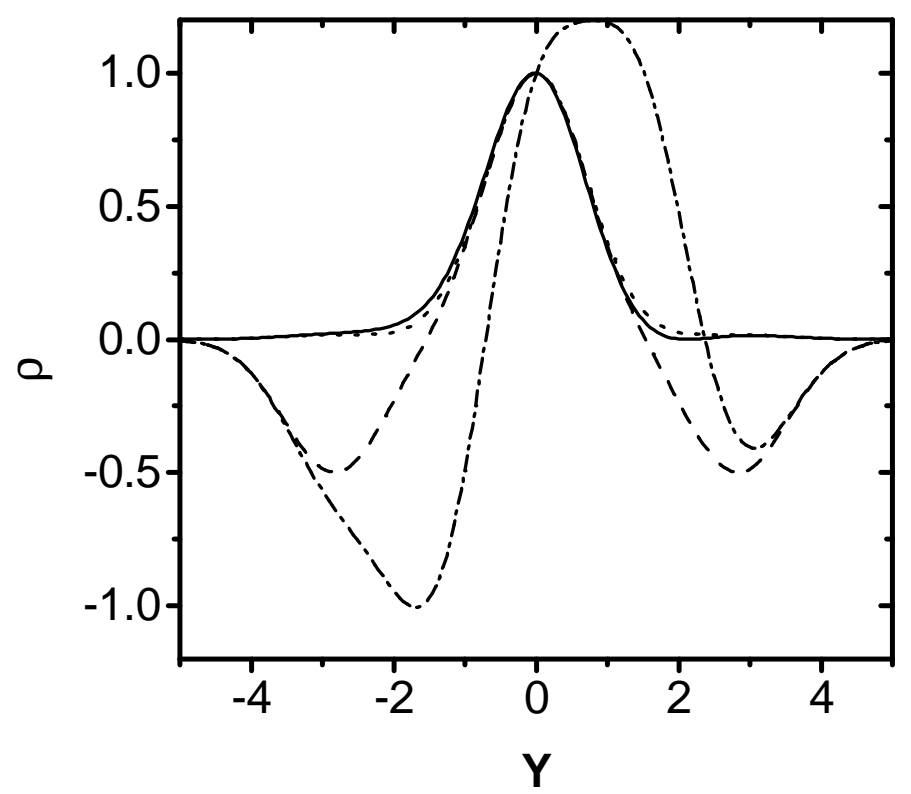

Fig. 3,

O. G. Balev et al.,

"Edge magnetoplasmons in modulated structures" 


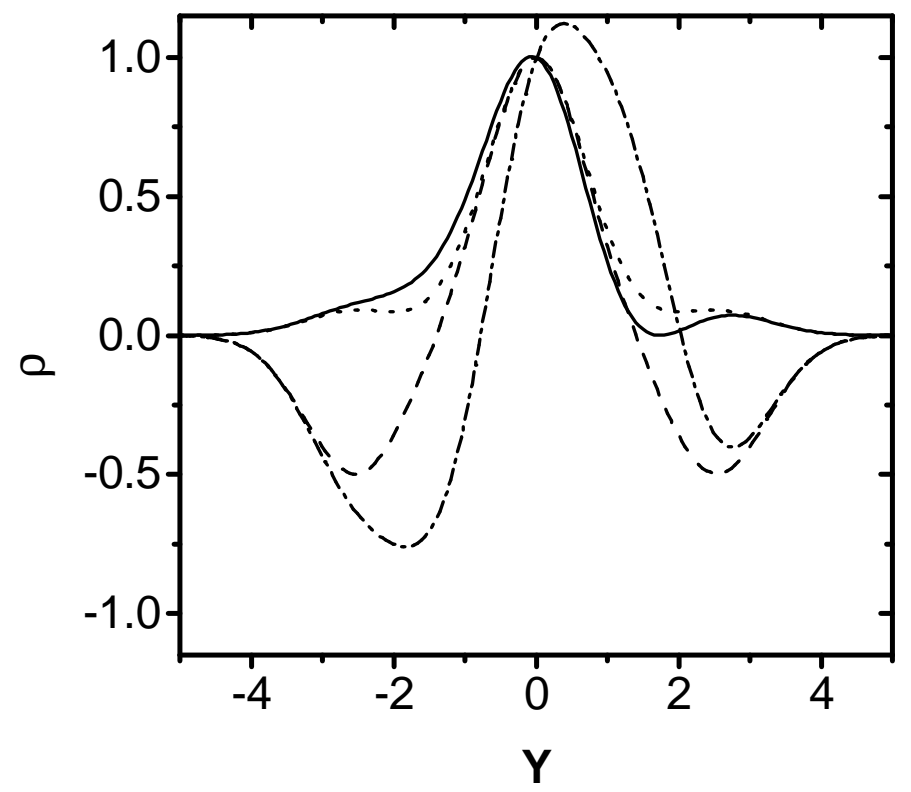

Fig. 4, O. G. Balev et al., "Edge magnetoplasmons in modulated structures" 